\title{
FrpA is the outer membrane piscibactin transporter in Vibrio anguillarum: structural elements in synthetic piscibactin analogues required for transport
}

\author{
Marta A. Lages ${ }^{1} \cdot$ M. Carmen de la Fuente ${ }^{2} \cdot$ Lucía Ageitos $^{2} \cdot$ Diana Martínez-Matamoros $^{2}$ (D) $\cdot$ Jaime Rodríguez $^{2}$ (D) \\ Miguel Balado $^{1}$ (D) . Carlos Jiménez ${ }^{2}$ (D) Manuel L. Lemos ${ }^{1}$ (D)
}

Received: 18 August 2021 / Accepted: 3 November 2021 / Published online: 18 November 2021

(C) The Author(s) 2021

\begin{abstract}
Piscibactin (Pcb) is a labile siderophore widespread among Vibrionaceae. Its production is a major virulence factor of some fish pathogens such as Photobacterium damselae subsp. piscicida and Vibrio anguillarum. Although FrpA was previously suggested as the putative outer membrane transporter (OMT) for ferri-piscibactin, its role in piscibactin uptake was never demonstrated. In this work, we generated mutants of $V$. anguillarum defective in FrpA and analyzed their ability to use piscibactin as iron source. The results showed that inactivation of frpA completely disables piscibactin utilization, and the original phenotype could be restored by gene complementation, confirming that FrpA is the OMT that mediates ferri-Pcb uptake. Additionally, the ability of several Pcb thiazole analogues, with different configurations at positions 9, 10, and 13, to be internalized through FrpA, was evaluated measuring their ability to promote growth under iron deficiency of several indicator strains. The results showed that while those analogues with a thiazole ring maintain almost the same activity as $\mathrm{Pcb}$, the maintenance of the hydroxyl group present in natural piscibactin configuration at position $\mathrm{C}-13$ is crucial for Fe ${ }^{3+}$ chelation and, in consequence, for the recognition of the ferri-siderophore by the cognate OMT. All these findings allowed us to propose a Pcb analogue as a good candidate to vectorize antimicrobial compounds, through the Trojan horse strategy, to develop novel compounds against bacterial fish diseases.
\end{abstract}

\section{Graphical abstract}

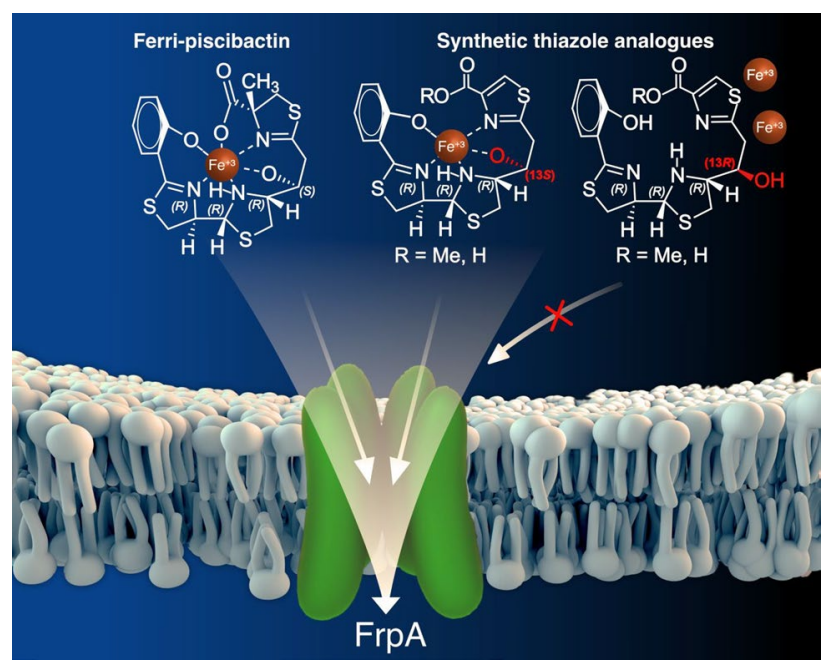

Keywords Vibrio anguillarum $\cdot$ Photobacterium damselae subsp. piscicida $\cdot$ Siderophores $\cdot$ Piscibactin $\cdot$ Fe(III)siderophore transporter

Extended author information available on the last page of the article 


\section{Introduction}

Bacteria belonging to Vibrionaceae family are present as free-living organisms in seawater, marine sediments, or colonizing marine organisms as symbionts. In addition, several pathogenic species for animals and humans are members of this family [1]. The spread of Vibrio spp. is favored by climate change and global seawater warming, which explains the increasing incidence of vibriosis in marine animals [2]. The emergence of novel diseases leads to a bottleneck effect that blocks the aquaculture sector from higher profit $[3,4]$. Although vaccination was shown as the most efficient way to minimize the incidence of infectious diseases [5], once outbreaks occur, the use of antibacterial compounds such as antibiotics is unavoidable. Unfortunately, their use increases the risk of drug resistance appearance in microbiota associated with fish farms and could also have adverse effects on consumers [6]. In this context, it is necessary the search of novel safe antimicrobials that can be effective against a wide range of bacterial pathogens.

The development of disease is closely associated with environmental signals that induce the expression of virulence factors [7]. Iron is essential for the survival and growth of almost all bacteria, but its availability is limited in the environment and within the host fluids [8]. In response to iron starvation, several pathogens have developed specific iron uptake mechanisms such as the synthesis and secretion of siderophores [9]. These iron chelators are secondary metabolites produced by most pathogenic bacteria to overcome iron starvation. Once the siderophore binds iron(III), the ferri-siderophore complex is acquired by bacteria through specific transporters [10]. Among the strategies aimed at developing novel antimicrobials, a promising approach called "Trojan Horse" consists in the use of siderophores conjugated to antimicrobial molecules which lead to a significantly increased antimicrobial activity, since they are using the high specific siderophore transport system to be internalized $[11,12]$. Using this strategy, Cefiderocol, a catechol-substituted siderophore cephalosporin, was recently approved for the treatment of aerobic Gram-negative bacteria infections in humans [13].

Piscibactin $(\mathrm{Pcb}, \mathbf{1})$ is a siderophore encoded by irp genes located in a high-pathogenicity island named irpHPI [14] and its production is a major virulence factor of some fish pathogens such as Photobacterium damselae subsp. piscicida (Pdp) [15] and Vibrio anguillarum (Va) [16], two of the most devastating bacterial pathogens in aquaculture worldwide. Notably, irp-HPI is widespread among Vibrionaceae [17]. A possible intermediate of Pcb, prepiscibactin (3) was also isolated from $P d p$ [15]. Thus, the piscibactin system could constitute a good candidate to vectorize antimicrobial compounds that could be used to develop novel antibacterials against the main bacterial fish diseases affecting aquaculture. Notably, the structure of Pcb (1) is very similar to that of yersiniabactin (2), the siderophore produced by some Yersina species such as $Y$. pestis and $Y$. enterocolitica, causative agents of severe enteric disorders in humans [18].

Besides piscibactin (1), two other siderophores have been described in $V$. anguillarum: vanchrobactin (4)[19] and anguibactin (5)[20] (Fig. 1). The high-pathogenicity island irp-HPI encodes functions related to piscibactin biosynthesis (irp123459), ferri-piscibactin transport (frpABC) and regulation of the system ( $\mathrm{araCl}$ and $\operatorname{araC2}$ ) (Fig. 2) $[14,15]$. A synthesis pathway was proposed based on the domain organization of biosynthetic enzymes [14]. Although according to amino acid sequence similarities the outer membrane protein FrpA, encoded by frpA gene in irp-HPI, should be the outer membrane transporter (OMT) of Pcb (1), its actual role in the ferric-siderophore internalization was never demonstrated. On the other hand, to rationally design vectors that exploit the "Trojan horse" strategy based on Pcb-conjugates, the transporter(s) involved in Pcb uptake must be characterized.

In this work, $V$. anguillarum was used as a model to study the role of FrpA in the uptake of the siderophore Pcb (1) by generating defective mutants of frpA gene. The results confirmed that FrpA is the outer membrane transporter that mediates ferri-Pcb uptake. Furthermore, the evaluation of the ability of several thiazole-Pcb analogues (6-9) to act as $\mathrm{Fe}^{3+}$ chelators and to be internalized via FrpA allowed us to determine structure-activity relationships (SAR) that would be essential for recognition of ferri-Pcb complex by its cognate transporter FrpA. The obtained results will be valuable for the preparation of conjugates, such as fluorescent probes or novel antimicrobials against piscibactin-synthesizing bacterial pathogens.

\section{Materials and methods}

\section{Bacterial strains, plasmids, and media}

Bacterial strains and plasmids used in this work are listed in Table S1. $V$. anguillarum and $P$. damselae subsp. piscicida strains were grown at $25{ }^{\circ} \mathrm{C}$ or $15{ }^{\circ} \mathrm{C}$ in Tryptic soy agar (TSA-1) or broth (TSB-1) supplemented with $1 \% \mathrm{NaCl}$. Escherichia coli strains were grown at $37{ }^{\circ} \mathrm{C}$ in Luria-Bertani (LB) agar or broth. When required, the medium was supplemented with antibiotics at the correspondent final concentration of: ampicillin sodium salt (Amp) at $60 \mu \mathrm{g} \mathrm{mL}^{-1}$ or $100 \mu \mathrm{g} \mathrm{mL}^{-1}$, kanamycin (Kan) at $50 \mu \mathrm{g} \mathrm{mL}^{-1}$, and gen-

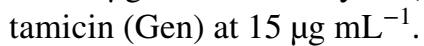


Fig. 1 Structures of the three siderophores, piscibactin (1), vanchrobactin (4), and anguibactin (5), involved in the iron (III) uptake in Vibrio anguillarum, yersiniabactin (2), prepiscibactin (3), and some of the thiazole-Pcb analogues, compounds 6-9, used in this work (the synthesis of these analogues will be published elsewhere)<smiles>[R]C([R])(C1=N[C@@](C)(C(=O)O)CS1)[C@@H](O)[C@H]1CS[C@H]([C@@H]2CSC(c3ccccc3O)=N2)N1</smiles>

1, $\mathrm{R}=\mathrm{H}$

2, $\mathrm{R}=\mathrm{Me}$<smiles>NC(=[NH2+])NCCC[C@H](NC(=O)c1cccc(O)c1O)C(=O)N[C@@H](CO)C(=O)[O-]</smiles><smiles>[R20]Oc1csc(C[C@H](O)[C@@H]2CS[C@@H]([C@H]3CSC(c4ccccc4O)=N3)N2)n1</smiles>

6a, $\mathrm{R}=\mathrm{Me}$

$7 a, R=H$<smiles></smiles>

3

5<smiles>[R20]Oc1csc(C[C@@H](O)[C@@H]2CS[C@H]([C@H]3CSC(c4ccccc4O)=N3)N2)n1</smiles>

epimers at C9 and C10

8a-d, $R=M e$

9a-d, $R=H$

Fig. 2 Genetic map of irp gene cluster encoding piscibactin (Pcb) biosynthesis and transport

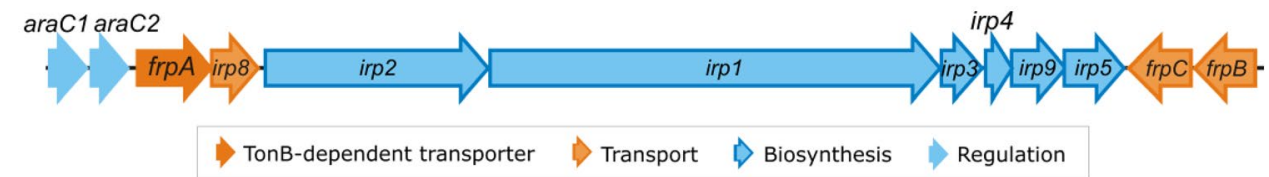

\section{Construction of frpA defective mutants by allelic exchange}

In frame deletions of the gene $f r p A$ were constructed by allelic exchange in $V$. anguillarum strains RV22 $\Delta v a b F$ (unable to synthesize vanchrobactin) and RV22 $\Delta v a b D$ (unable to synthesize both vanchrobactin and piscibactin). The flanking regions of $\operatorname{frp} A$ were amplified by PCR and cloned into the high copy vector $\mathrm{pWKS} 30$. The construction was ligated into the suicide vector pNidKan [21]. The resulted plasmid was conjugated with the previously mentioned strains and the selection was performed based on kanamycin resistance. After a second recombination event, the mutant strains were selected based on sucrose resistance (15\%), and the consequent plasmid loss was confirmed by screening the growth on kanamycin and ampicillin plates. Subsequently, a
PCR was performed to confirm the allelic exchange. This led to the generation of the mutant strains RV22 $\triangle v a b F \Delta f r p A$ and RV22 $\Delta v a b D \Delta f r p A$. For the complementation of the gene, frpA was amplified by PCR and cloned into the vector pSEVA651 in E. coli S17-1 $\lambda$ pir. The plasmid was mobilized into the mutant strains to restore the original phenotypes. The oligonucleotides used are listed in Table S2.

\section{Growth ability and siderophore production under iron-limiting conditions}

Overnight cultures of $V$. anguillarum RV22 $\Delta v a b F$, RV22 $\Delta v a b F \Delta f r p A, \mathrm{RV} 22 \Delta v a b D$, and RV22 $\Delta v a b D \Delta$ frpA were made in TSB-1. The $\mathrm{OD}_{600}$ was adjusted to 0.5 and a 1:50 dilution was inoculated in $5 \mathrm{~mL}$ of CM9 medium. Each culture was supplemented with $10 \mu \mathrm{M} \mathrm{FeCl}_{3}$, for iron excess 
conditions or with 50 or $75 \mu \mathrm{M} 2,2^{\prime}$-dipyridyl to achieve the iron-deficient conditions. The cultures were incubated at $15{ }^{\circ} \mathrm{C}$, with shaking at $120 \mathrm{rpm}$. After $48 \mathrm{~h}$, the final growth was recorded in a spectrophotometer (Hitachi). Additionally, when the cultures reached an $\mathrm{OD}_{600} \approx 0.8$, they were centrifuged at $10,000 \mathrm{rpm}$ and the supernatant was used to perform a chrome azurol-S (CAS) liquid assay [22]. The supernatant was mixed 1:1 with the CAS reagent. After 15 min of incubation at room temperature, the $A_{630}$ was measured in a spectrophotometer (Hitachi).

\section{Isolation of Piscibactin-Fe(III) complex from Vibrio anguillarum RV22 $\Delta$ vabF}

Using the procedure describes by Souto et al. [15], the isolation of piscibactin-Fe(III) was carried out as follows: $1 \mathrm{~L}$ of centrifuged cell-free culture broth of Vibrio anguillarum RV22 $\Delta v a b F$ was treated with $17 \mathrm{mg}$ of $\mathrm{FeCl}_{3} \cdot 6 \mathrm{H}_{2} \mathrm{O}$ and then concentrated in vacuo to $0.30 \mathrm{~L}$. The sample was passed through four OASIS ${ }^{\circledR}$ HLB cartridges $\left(35 \mathrm{~cm}^{3}, 6 \mathrm{~g}\right)$, eluted with $30 \mathrm{~mL}$ of the following mixtures of $\mathrm{H}_{2} \mathrm{O}$ and $\mathrm{CH}_{3} \mathrm{CN}$ : $1: 0,7: 3,1: 1,3: 7,0: 1$, and the fraction eluted with a mixture of $\mathrm{H}_{2} \mathrm{O}$ and $\mathrm{CH}_{3} \mathrm{CN}$ (1:1) was collected. This fraction was concentrated under vacuum to about $5 \mathrm{~mL}$ under reduced pressure and submitted to RP-HPLC. Finally, separation was achieved using a Discovery ${ }^{\circledR}$ HS F5 $(100 \times 4.6 \mathrm{~mm}, 5 \mu \mathrm{m})$ column (Supelco) with a 5 min gradient from 10 to $20 \%$ of $\mathrm{CH}_{3} \mathrm{CN}$ in $\mathrm{H}_{2} \mathrm{O}$, a 10 min isocratic period at $20 \%$ of $\mathrm{CH}_{3} \mathrm{CN}$ in $\mathrm{H}_{2} \mathrm{O}$, a 5 min gradient from 20 to $100 \% \mathrm{CH}_{3} \mathrm{CN}$ in $\mathrm{H}_{2} \mathrm{O}$ and finally a $5 \mathrm{~min}$ isocratic period at $100 \%$ of $\mathrm{CH}_{3} \mathrm{CN}$ at a flow rate of $1 \mathrm{~mL} / \mathrm{min}$ (UV detector operating at $\lambda=264 \mathrm{~nm}$ ). Fractions containing piscibactin-Fe(III) complex (100\% of $\mathrm{CH}_{3} \mathrm{CN}$ in $\mathrm{H}_{2} \mathrm{O}, R_{t}=20 \mathrm{~min}$ ) were pooled and dried under vacuum. This procedure provided ca. $1 \mathrm{mg}$ of piscibactin$\mathrm{Fe}(\mathrm{III})$ complex.

\section{Cross-feeding assays}

Cross-feeding assays were performed to analyze whether several $V$. anguillarum strains could use piscibactin as iron source. $1 \mathrm{~mL}$ of a TSB- 1 culture at an $\mathrm{OD}_{600} \approx 0.8$ of the indicator strains, RV22 $\Delta v a b F$ and RV22 $\Delta v a b F \Delta f r p A$, was inoculated into $20 \mathrm{~mL}$ of CM9 minimal medium containing $0.8 \%$ agarose and supplemented with $90 \mu \mathrm{M} 2,2^{\prime}$-dipyridyl and poured onto plates. The strains to be tested for piscibactin production RV22 $\Delta v a b F$ (piscibactin producer), RV22 $\triangle v a b D$ (unable to produce piscibactin and vanchrobactin), and RV22 $\Delta v a b F \Delta$ frpA were cultured in TSA-1 plates supplemented with $50 \mu \mathrm{M} 2,2^{\prime}$-dipyridyl, and a loopful of biomass was harvested and placed onto the surface of the plates previously inoculated with the indicator strain. After $48 \mathrm{~h}$ incubation at $25^{\circ} \mathrm{C}$, a growth halo around the tested strains indicated the utilization of the siderophore.

\section{Biological activity of piscibactin analogues}

The biological activity of piscibactin analogues, $\mathbf{6 - 9}$, was evaluated in 96-well microtiter plates, using $200 \mu \mathrm{L}$ as final volume. To determine the lowest concentration that allows growth, piscibactin and each analogue were used at the final concentrations of $20,10,2$, and $0.2 \mu \mathrm{M}$ from a stock solution prepared with methanol:milliQ water $(1: 1)$. From an overnight culture of RV22 $\Delta v a b D, \operatorname{RV} 22 \Delta v a b D \Delta f r p A$ and $P$. damselae subsp. piscicida, whose $\mathrm{OD}_{600}$ was adjusted to 0.5 , a final dilution of 1:20 and 1:40, respectively, was inoculated in CM9 media supplemented with $75 \mu \mathrm{M}$ 2,2'-dipyridyl. After the addition of piscibactin and the tested analogues at the suitable concentrations, the plate was incubated at $25^{\circ} \mathrm{C}$ with shaking at $120 \mathrm{rpm}$. The growth $\left(\mathrm{OD}_{600}\right)$ was recorded for $18 \mathrm{~h}$ in an iMACK Microplate reader (Bio-Rad). Media supplemented with ferric chloride $\left(10 \mu \mathrm{M} \mathrm{FeCl}_{3}\right)$, with the chelating agent $2,2^{\prime}$-dipyridyl $(75 \mu \mathrm{M})$, and non-inoculated media were used as controls. The assay corresponding to each condition (iron excess or iron deficiency) and each bacterial strain was performed in duplicate in each plate. Three independent experiments were performed, and the results shown are the mean of the values obtained.

\section{FrpA phylogenetic analysis}

To analyze the diversity of piscibactin outer membrane receptor FrpA, we performed BlastN searches in the nucleotide collection (nr/nt) and whole-genome shotgun (wgs) NCBI databases using as a query frpA nucleotide sequence of $V$. anguillarum RV22. frpA homologs were downloaded from NCBI and aligned using MUSCLE (MEGA 6 suite). The phylogenetic tree was constructed by the neighbor-joining method with a bootstrap method of 1000 replicates using also MEGA 6 software.

\section{Results and discussion}

\section{Inactivation of frpA disables Pcb utilization as iron source}

The irp-HPI island contains genes $(\operatorname{frp} A B C)$ that are predicted to encode the ferri-piscibactin uptake machinery (Fig. 2) [16]. Particularly, frpA gene would encode the putative outer membrane transporter of the ferri-piscibactin complex. To confirm the role of FrpA in Pcb uptake, an in-frame deletion mutant for this gene was obtained using $V$. anguillarum RV22 $\triangle v a b F$ as parental strain, a strain that only synthesizes Pcb (1) and not vanchrobactin. The ability of the generated mutant to use Pcb (1) as iron source was first studied by its ability to grow under iron deficiency. Strain RV22 $\Delta v a b D$, a mutant unable to synthesize neither 
Pcb (1) nor vanchrobactin (4), and thus unable to grow under iron deficiency, was used as negative control. As shown in

$\mathrm{CM} 9+\mathrm{FeCl}_{3} 10 \mu \mathrm{M} \square \mathrm{CM} 9+2,2-$ Dipyridyl $75 \mu \mathrm{M} \quad \square$ CAS assay

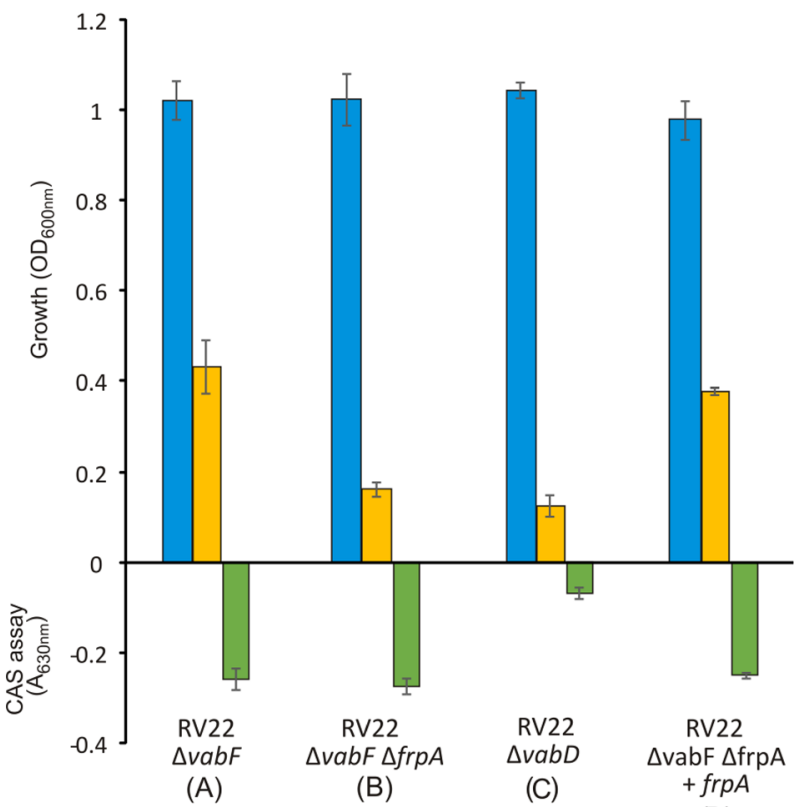

(D)

Fig. 3 Growth under high or low iron conditions and siderophore production measured by CAS assay of parental strain (A) V. anguillarum RV22 $\Delta v a b F$, its derivative $\Delta$ frpA mutant (B) (RV22 $\Delta v a b F \Delta f r p A)$, and control strain (C) (RV22 $\Delta v a b D)$ that is unable to synthetize any siderophore. The frpA complemented strain (D) was obtained by the introduction of the wild-type frpA gene, cloned in the plasmid pSEVA, into $V$. anguillarum $\triangle$ frpA mutant
Fig. 3, while the parental strain RV22 $\triangle v a b F$ could grow under high iron deficiency (CM9 containing 2,2'-dipyridyl at $75 \mu \mathrm{M}$ ), the frpA defective mutant (RV22 $\triangle v a b F \Delta$ frpA) was totally impaired to grow under these conditions. Complementation of the frpA defective mutant with a copy of the wild-type gene restored the growth phenotype at the same levels as the parental strain (Fig. 3). These results clearly indicate that FrpA must act as the OMT for Pcb (1).

In addition, the evaluation of siderophore activity in supernatants by the CAS assay showed that the mutant RV22 $\Delta v a b F \Delta f r p A$ produces the same siderophore levels as its parental strain RV22 $\Delta v a b F$ (Fig. 3) and that it was able to cross-feed RV22 $\Delta v a b F$ (Fig. 4). However, RV22 $\triangle v a b F \Delta f r p A$ could not be cross-fed by any piscibactin-producer strain, except by the wild-type RV22 due to the utilization of vanchrobactin. Thus, cross-feed assays confirm that the mutant defective in FrpA outer membrane transporter (RV22 $\Delta v a b F \Delta f r p A)$ was impaired to use Pcb (1) as iron source (Fig. 4).

The results obtained in the grow cross-feeding assays greatly suggest that FrpA has an active role in the ability of RV22 $\Delta v a b F$ to grow in iron-limited conditions and that it must be involved in the internalization of ferri-piscibactin, acting as the OMT for this siderophore.

Outer membrane transporters of siderophores are generally considered to be quite specific for their cognate ligand [23]. However, some works identified siderophore transporters that can transport multiple related ligands [24-27]. One of the most versatile siderophore transporters described to date is FstC, the amonabactin OMT of A. salmonicida [27]. FstC mediates the uptake of the four natural amonabactin forms and also internalizes some biscatecholate amonabactin

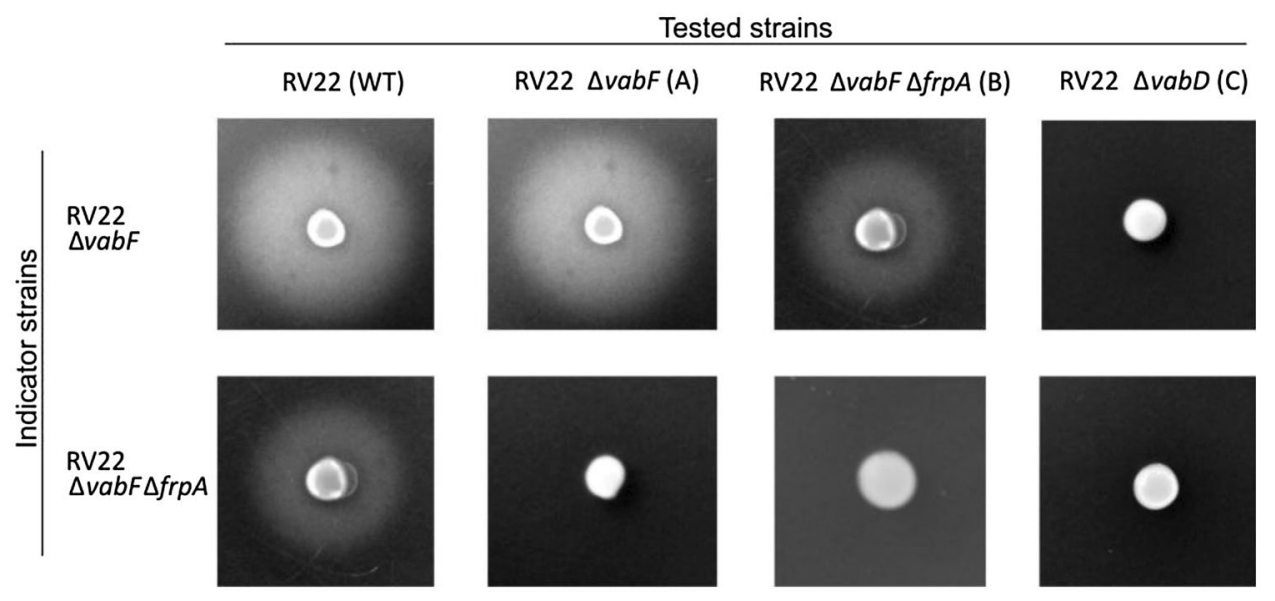

Fig. 4 Cross-feeding assay to analyze the utilization of Pcb (1) by an FrpA mutant. RV22 $\triangle v a b F$ [producing Pcb (1) but unable to produce vanchrobactin] and RV22 $\Delta v a b F \Delta f r p A$ [producing Pcb (1) but potentially unable to utilize it] were used as indicator strains. Wild-type strain RV22 (WT) and its derivative mutants RV22 $\Delta v a b F(\mathbf{A})$, RV22
$\Delta v a b F \Delta f r p A(\mathbf{B})$ and RV22 $\Delta v a b D(\mathbf{C})$ (unable to synthesize any siderophore), were used as tested strains. A growth halo of the indicator strains around the tested strains evidences that they can use the siderophore produced by the tested strains to grow under iron-limited conditions 
analogues with rather different chemical structures [27]. The successful use of siderophore uptake mechanisms to vectorize compounds depends on the selection of a versatile target transporter and the characterization of the structures of the siderophore required for its recognition by the cognate OMT. Thus, once demonstrated that FrpA is the outer membrane transporter for piscibactin, some piscibactin analogues were synthesized and analyzed for their ability to be internalized by FrpA.

\section{Evaluation of the siderophore activity of Pcb (1) and Pcb thiazole analogues 6-9 in V. anguillarum}

Since the presence of the acid sensitive $\beta$-hydroxy-2,4disubstituted thiazoline moiety [28] in $\mathrm{Ga}^{+3}-\mathrm{Pcb}$ complex $\left(1-\mathrm{Ga}^{+3}\right)$ represented a challenge in our reported total synthesis for this compound [29], we have found that the substitution of this thiazoline ring for a thiazole ring that is less sensitive to acids, due to its aromatic character, facilitated the synthesis of analogues (manuscript in preparation).

In this way, two sets of Pcb analogues bearing a thiazole ring (6-9) but differing in the configuration of the hydroxy group at C-13 position (Fig. 5) were previously synthetized and then evaluated with the aim of determining some structural requirements for Pcb (1) recognition by its OMT protein FrpA and searching for a simplified Pcb analogue that keeps the siderophore activity.

The biological activity of synthetic Pcb thiazole analogues 6-9 was compared to the natural Pcb (1) using growth promotion assays against $\operatorname{FrpA}(+)(\mathrm{RV} 22 \Delta v a b D)$ and $\operatorname{FrpA}(-)(\mathrm{RV} 22 \Delta v a b D \Delta f r p A)$ strains (Fig. 6). Since inactivation of $v a b D$ abolishes siderophore production, both vanchrobactin and piscibactin [16], the growth achieved by these strains will be proportional to the bio-availability of the Pcb or analogue 6-9 tested. Pcb (1) efficiently promoted, in iron-limiting conditions, the growth of $V$. anguillarum RV22 $\Delta v a b D$ mutant. For each concentration tested, Pcb (1) showed the highest siderophore activity of all compounds tested. As expected, Pcb (1) was unable to restore the growth of the FrpA(-) defective mutant, which confirms that FrpA mediates the iron uptake of $\mathrm{Pcb}(\mathbf{1})$ in $V$. anguillarum RV22 (Fig. 6). Notably, addition of Pcb (1) in the range 2-20 $\mu \mathrm{M}$ increased the maximum growth achieved (Fig. 6), being indistinguishable from that observed when ferric chloride was added to the medium. Results of growth promotion assays showed that all $13 S$ thiazole-Pcb analogues, compounds 6 and 7, stimulate the growth of $\operatorname{FrpA}(+)$ at all concentrations tested. However, they were less effective than endogenous Pcb (1). On the contrary, 13R thiazole analogues $\mathbf{8}$ and $\mathbf{9}$ with opposite configuration at position C-13 did not support growth of neither $\operatorname{FrpA}(+)$ nor $\operatorname{FrpA}(-)$ (Fig. 6).

Chrome azurol-S (CAS) liquid assay was used to assess the ability of each analogue to chelate iron [22]. Results showed that Pcb (1) and the $13 S$ thiazole-Pcb analogues 6 and $\mathbf{7}$ gave a positive CAS test, which showed that these compounds $(\mathbf{1}, \mathbf{6}$, and 7) chelate iron. By contrast, $13 R$ thiazole-Pcb analogues $\mathbf{8}$ and $\mathbf{9}$ displayed a negative result in the CAS assay. These findings are in agreement with unsuccessful $\mathrm{Ga}^{3+}$ complexation attempts with the $13 R$ acid thiazole analogues 8 . Additionally, although the mixture of thiazole-Pcb analogues $\mathbf{7 b}$,c exhibited a slight higher biological activity than their corresponding methyl esters $\mathbf{6 b}, \mathbf{c}$<smiles>[R20]C(=O)c1csc(C[C@H](O)[C@H]2CS[C@@H]([C@H](O)[C@@H]3CSC(c4ccccc4O)=N3)N2)n1</smiles>
6a, $\mathrm{R}=\mathrm{H}$
$7 \mathrm{a}, \mathrm{R}=\mathrm{Me}$
$6 b, R=H$
$7 b, R=M e$

$6 \mathrm{c}, \mathrm{R}=\mathrm{H}$

7c, $\mathrm{R}=\mathrm{Me}$

6d, $\mathrm{R}=\mathrm{H}$

7d, $\mathrm{R}=\mathrm{Me}$<smiles>[R20]C([R20])c1csc(C[C@H](O)[C@@H]2CS[C@@H]([C@H]3CSC(c4ccccc4O)=N3)N2)n1</smiles><smiles>[R20]Oc1csc(C[C@H](O)[C@H]2CS[C@H](c3csc(-c4ccccc4O)n3)N2)n1</smiles>

$8 \mathbf{a}, \mathrm{R}=\mathrm{H}$
$9 \mathbf{a}, \mathrm{R}=\mathrm{Me}$

8b, $R=H$

9b, $\mathrm{R}=\mathrm{Me}$

$8 c, R=H$

9c, $\mathrm{R}=\mathrm{Me}$

8d, $\mathrm{R}=\mathrm{H}$

9d, $\mathrm{R}=\mathrm{Me}$

Fig. 5 Structure of the synthetic Pcb thiazole analogues 6-9 (manuscript in preparation) evaluated in this work 


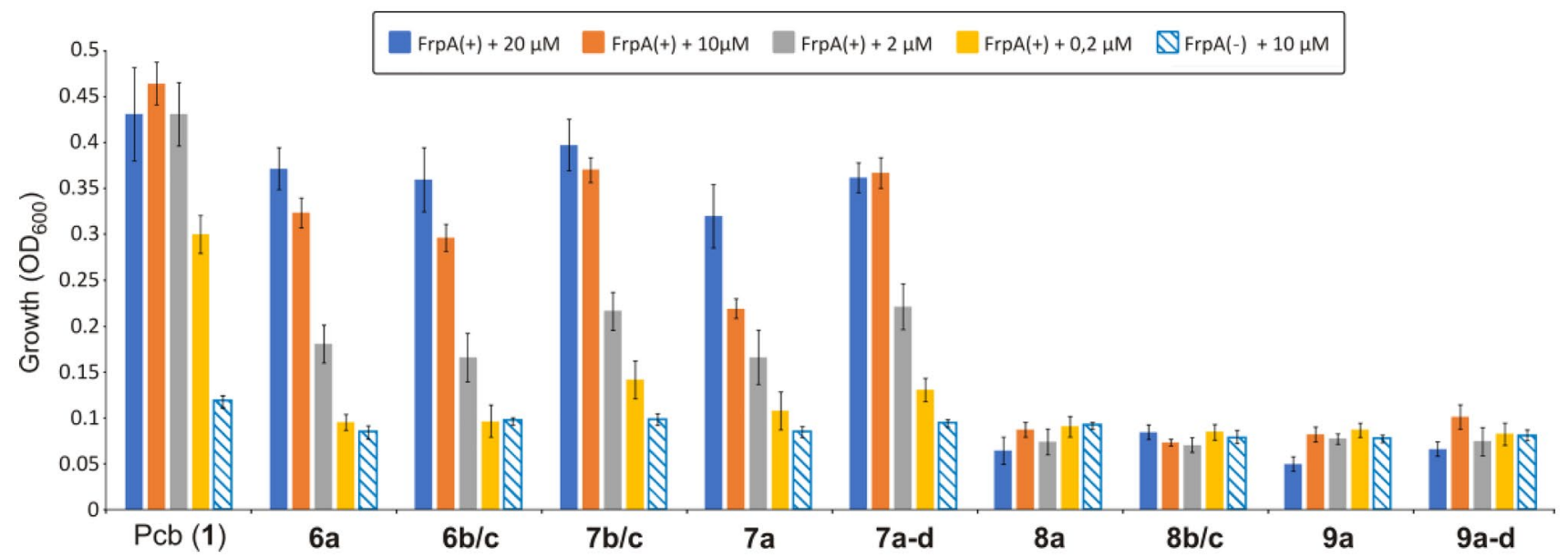

Fig. 6 Biological activity (maximum growth achieved) of Pcb (1) and thiazole-Pcb analogues 6-9 measured by growth promotion of a $V$. anguillarum $\operatorname{FrpA}(+)$ strain, carrying a functional frpA gene (RV22 $\Delta v a b D)$, or an $\operatorname{FrpA}(-)$ strain (RV22 $\Delta v a b D \Delta f r p A)$. The assays

and thiazole-Pcb analogue 7a, the differences were not statistically significant. In the same way, similar activities were obtained for $13 S$ thiazole-Pcb analogues 6 and 7 which suggest that the configuration at positions $\mathrm{C}-9$ and $\mathrm{C}-10$ has no major influence on the biological activity of these analogues. All results put together greatly suggest that the configuration of the hydroxyl group at C-13 is crucial for the chelation of $\mathrm{Fe}^{3+}$ and, in consequence, for the molecular recognition by the outer membrane transporter FrpA. Most notably, the siderophore activity showed by the $13 S$ thiazole analogues 6 and $\mathbf{7}$ indicates that the substitution of the thiazoline ring for a thiazole ring and/or the presence of a methyl ester group instead of a carboxylic acid functionality does not affect to the recognition of Pcb analogues by FrpA. The possible presence of esterases during the uptake process could explain the similar biological activity of the methyl esters in relation to their corresponding acids. These results suggest that these groups do not constitute key structural features of the siderophore and likely they do not interact in a distinctive way with the recognition domain of the FrpA transporter.

Pcb (1) and yersiniabactin (2) share structural characteristics and their labile nature is a disadvantage in terms of chemical synthesis and manipulation of the molecule [15, 30]. Pcb (1) can be stabilized by chelation with iron(III) or gallium(III) [15]. As previously described, Pcb (1) chelates gallium(III) through the three nitrogens and the three oxygens present in the siderophore [15]. Our result showed that configuration at position $\mathrm{C}-13$ is crucial for metal chelation and, consequently, its alteration disables the biological activity of the siderophore.

Taking into account that the substitution of the thiazoline ring in Pcb (1) for a thiazole ring facilitated the synthesis of were performed in CM9 minimal medium supplemented with $75 \mu \mathrm{M}$ 2,2'-dipyridil and one of the compounds to be tested at concentrations between 0.2 and $20 \mu \mathrm{M}$. Error bars represent standard deviation

Pcb analogues and the fact that their methyl ester derivatives efficiently promote the growth of $\operatorname{FrpA}(+)$ bacterial strains (Fig. 6), these type of analogues could be good candidates to be used as vectors for future conjugation with antimicrobials or fluorescent probes following the "Trojan horse strategy".

\section{Phylogenetic analysis of frpA and siderophore activity in $P$. damselae subsp. piscicida}

Piscibactin siderophore system is encoded by a genomic island (irp-HPI) that is widespread among Vibrionaceae, including species grouped in the Harveyi and Splendidus clades $[16,17,21,31]$. Thus, some different versions (homologues) of the frpA gene encoding the piscibactin transporter FrpA are present in different bacterial species. A phylogenetic analysis with those frpA versions available in GenBank was performed. The resultant phylogenetic tree is shown in Fig. 7. The results showed that frpA is widespread not only in Vibrionaceae, but also it is present in many species of gamma-proteobacteria like Shewanella, Marinomonas and enterobacteria like Xenorhabdus, Photorhabdus, and Providencia with similarities higher than $62 \%$. V. anguillarum $\operatorname{frpA}\left(\operatorname{frp} A_{\text {Vang }}\right)$ is clustered in a clade with $V$. ordalii and $V$. qinghaiensis. In addition, these $f r p A$ sequences are clustered with a bootstrap value of 100 with several $V$. cholerae sequences, which reflect a close relationship between the frpA homologues present in the genome of these bacteria.

The outer membrane transporter FrpA of $V$. anguillarum $\left(\mathrm{FrpA}_{\text {Vang }}\right)$ shares a $66 \%$ homology identity and $81 \%$ similarity with the version of FrpA present in the genome of P. damselae subsp. piscicida $\left(\operatorname{FrpA}_{P d p}\right)$. Gene $f r p A_{P d p}$ is clustered with Vibrio species generally related to bivalve 
Fig. 7 Phylogenetic tree of $\operatorname{frpA}$ gene homologues encoding the Pcb outer membrane transporter FrpA found in GenBank (black). Labels denote species name, accession number, and number of identical genes deposited in GenBank. The tree is drawn to scale and branch lengths denote nucleotide p-distance (p-distance method). Bootstrap values higher than $70 \%$ are shown next to the branches. All positions containing gaps and missing data were eliminated. There was a total of 611 positions in the final dataset

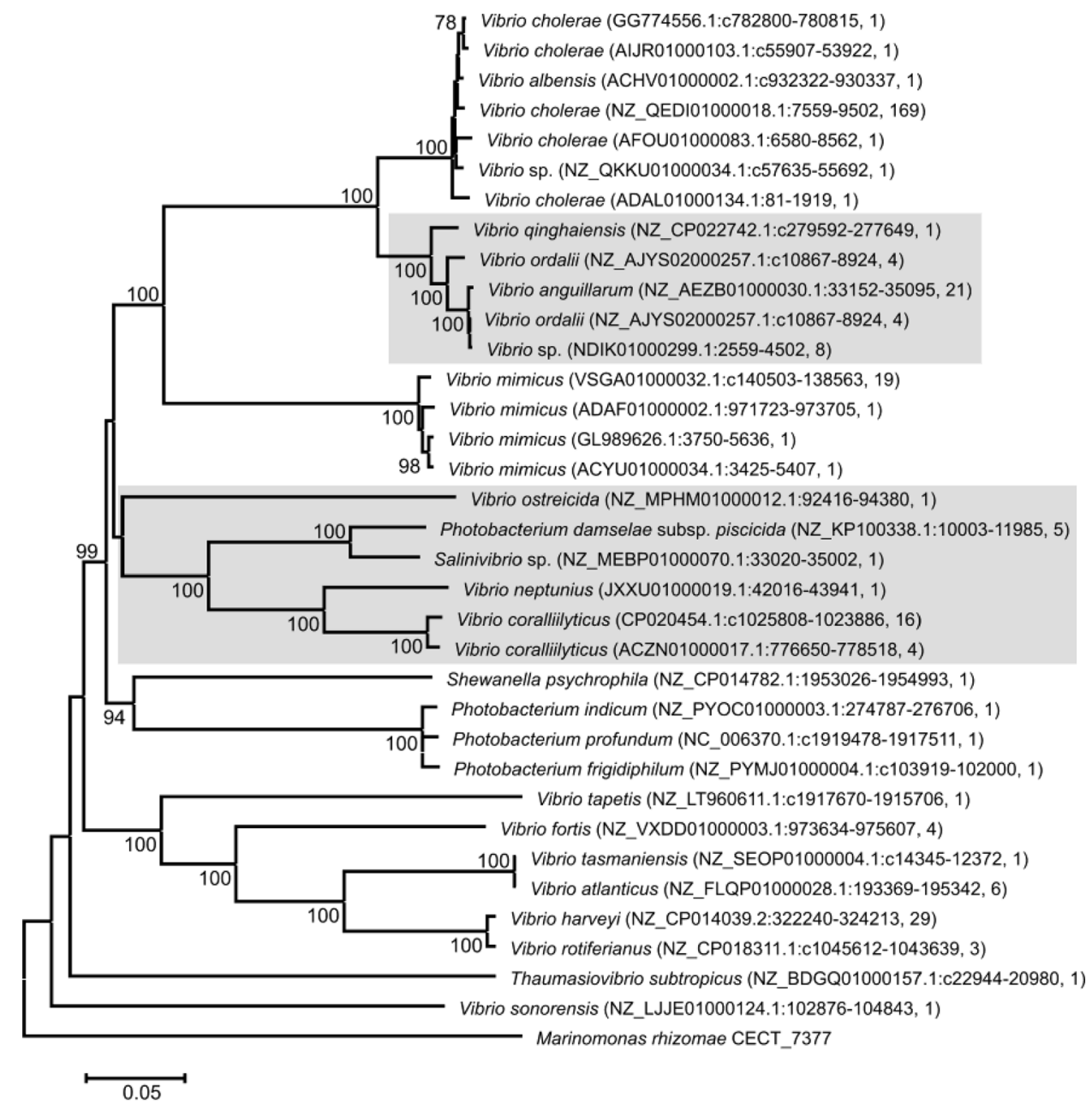

molluscs infections such as $V$. ostreicida, $V$. coralliilyticus, and V. neptunius [32].

Additionally, growth promotion assays against $P d p$ DI21 strains were used to study the biological activity of Pcb (1) and synthetic Pcb thiazole analogues $\mathbf{6}$ and $\mathbf{7}$ in these species (Fig. 8). The results showed that $13 S$ piscibactin analogues are also internalized by $P$. damselae subsp. piscicida via $\operatorname{FrpA}_{P d p}$, and also suggest that this transporter displays almost the same specificity for the synthetic $13 S$ Pcb analogues 6 and $\mathbf{7}$ than for Pcb (1). All results together greatly suggest that the activity of piscibactin analogues synthesized here could be used not only to vectorize compounds against $V$. anguillarum but also against $P d p$ and those other bacterial species that carry irp-HPI like elements in their genome.

\section{Conclusion}

The recognition of iron acquisition systems mediated by siderophores as relevant bacterial virulence factors and the fact that they are essential for the survival and growth of pathogens inside their hosts make them excellent

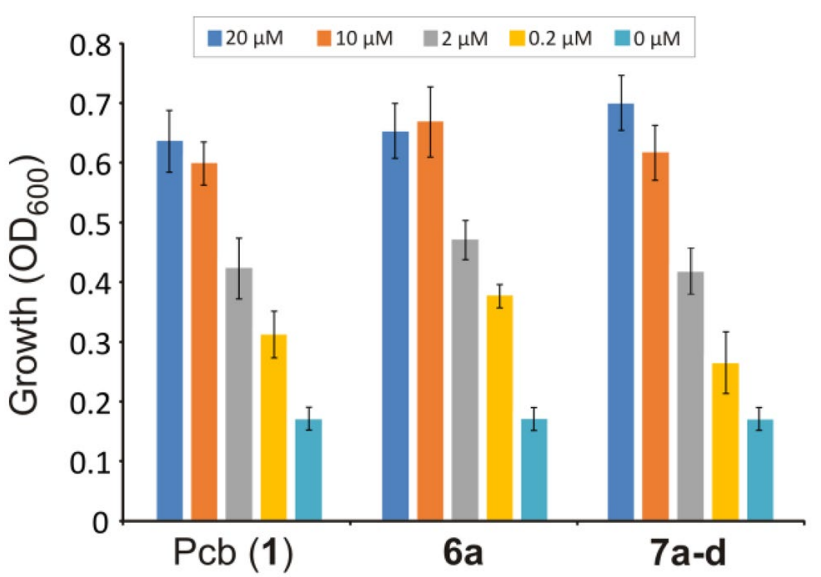

Fig. 8 Utilization as iron source (maximum growth achieved) of Pcb (1) and the synthetic piscibactin analogues $\mathbf{6 a}$ and 7 by $P$. damselae subsp. piscicida DI21 carrying a functional FrpA

candidates for the development of novel antimicrobial strategies. Pcb system is widespread among diverse marine bacterial pathogens and, consequently, it could be used 
as a target to prevent the occurrence of some infectious diseases. In this regard, it has been previously shown that FrpA $_{P d p}$, the piscibactin transporter of $P$. damselae subsp. piscicida, could be used as an immunogenic protein to formulate vaccines against fish photobacteriosis [33]. Alternatively, a different application could be based on the use of siderophore conjugates to vectorize antimicrobials following a Trojan horse approach [11] or to design fluorescent probes that could be used in the early diagnosis [34].

In this work, FrpA was characterized as the TonBdependent transporter that mediates ferri-piscibactin uptake. Then, the evaluation of some synthetic Pcb analogues led to the definition of the siderophore chemical structures that enable ferri-piscibactin recognition by FrpA, its cognate outer membrane transporter. Our results showed that the presence of a thiazole ring instead of thiazoline scarcely affects the biological activity of piscibactin analogues. By contrast, the maintenance of the natural piscibactin configuration at position $\mathrm{C}-13$ is crucial for $\mathrm{Fe}^{3+}$ chelation and, therefore, for the recognition of the ferri-siderophore by the cognate OMT. Our results also demonstrated that Pcb thiazole analogues can be internalized by $V$. anguillarum and by $P d p$ via FrpA $\mathrm{A}_{\text {Vang }}$ and $\operatorname{FrpA}_{P d p}$, respectively. Hence, it is predicted that $\mathrm{Pcb}$ analogues conjugates could be active for a large diversity of irp-HPI-carrying bacteria such as the fish pathogens $V$. anguillarum and $P$. damselae subsp. piscicida; bivalve pathogens including V. ostreicida, V. neptunius, and V. coralliilyticus; and also strains of V. cholerae. All together, these findings allowed us to propose a Pcb analogue as a good candidate to vectorize antimicrobial compounds, through the Trojan horse strategy, to develop novel compounds against bacterial fish diseases.

Future studies will allow us to test the use of different conjugates based on piscibactin to develop novel antimicrobials or other compounds with diverse biotechnological applications, that can be efficiently transported into Vibrionaceae bacterial cells using FrpA outer membrane transporter.

Supplementary Information The online version contains supplementary material available at https://doi.org/10.1007/s00775-021-01916-1.

Acknowledgements This work was supported by grants RTI2018093634-B-C21/C22 (AEI/FEDER, EU), cofunded by the FEDER Programme from the European Union, and by Grant PID2019103891RJ-100 (AEI) from the Agency for Research (AEI) of Spain. Work in University of Santiago de Compostela and University of A Coruña was also supported by grants GRC2018/018 and GRC2018/039, respectively, from Xunta de Galicia and BLUEBIOLAB (0474_BLUEBIOLAB_1_E), Programme INTERREG V A of Spain-Portugal (POCTEP). L.A. thanks Xunta de Galicia (Spain) for a predoctoral fellowship (ED481A-2019/081) co-financed by European Social Fund (ESF).
Author contributions The manuscript was written through contributions of all authors. All authors have given approval to the final version of the manuscript.

Funding Open Access funding provided thanks to the CRUE-CSIC agreement with Springer Nature.

\section{Declarations}

Conflict of interest The authors declare that they have no known competing financial interests or personal relationships that could have appeared to influence the work reported in this paper.

Open Access This article is licensed under a Creative Commons Attribution 4.0 International License, which permits use, sharing, adaptation, distribution and reproduction in any medium or format, as long as you give appropriate credit to the original author(s) and the source, provide a link to the Creative Commons licence, and indicate if changes were made. The images or other third party material in this article are included in the article's Creative Commons licence, unless indicated otherwise in a credit line to the material. If material is not included in the article's Creative Commons licence and your intended use is not permitted by statutory regulation or exceeds the permitted use, you will need to obtain permission directly from the copyright holder. To view a copy of this licence, visit http://creativecommons.org/licenses/by/4.0/.

\section{References}

1. Igbinosa EO, Okoh AI (2008) Emerging Vibrio species: an unending threat to public health in developing countries. Res Microbiol 159:495-506. https://doi.org/10.1016/j.resmic.2008.07.001

2. Baker-Austin C, Oliver JD, Alam M et al (2018) Vibrio spp. infections. Nat Rev Dis Prim 4:8. https://doi.org/10.1038/ s41572-018-0005-8

3. Ackleh AS, Allen LJS (2003) Competitive exclusion and coexistence for pathogens in an epidemic model with variable population size. J Math Biol 47:153-168. https://doi.org/10.1007/ s00285-003-0207-9

4. Márquez I, García-Vázquez E, Borrell YJ (2014) Possible effects of vaccination and environmental changes on the presence of disease in northern Spanish fish farms. Aquaculture 431:118-123. https://doi.org/10.1016/J.AQUACULTURE.2013.12.030

5. Ji Q, Wang S, Ma J, Liu Q (2020) A review: progress in the development of fish Vibrio spp. vaccines. Immunol Lett 226:46-54. https://doi.org/10.1016/j.imlet.2020.07.002

6. Klase G, Lee S, Liang S et al (2019) The microbiome and antibiotic resistance in integrated fishfarm water: implications of environmental public health. Sci Total Environ 649:1491-1501. https://doi.org/10.1016/J.SCITOTENV.2018.08.288

7. Dandekar T, Eisenreich W (2015) Host-adapted metabolism and its regulation in bacterial pathogens. Front Cell Infect Microbiol 5:28. https://doi.org/10.3389/fcimb.2015.00028

8. Lemos ML, Balado M (2020) Iron uptake mechanisms as key virulence factors in bacterial fish pathogens. J Appl Microbiol. https://doi.org/10.1111/jam.14595

9. Ratledge C, Dover LG (2000) Iron metabolism in pathogenic bacteria. Annu Rev Microbiol 54:881-941. https://doi.org/10.1146/ annurev.micro.54.1.881

10. Hider RC, Kong X (2010) Chemistry and biology of siderophores. Nat Prod Rep 27:637-657. https://doi.org/10.1039/b906679a 
11. Schalk IJ (2018) Siderophore-antibiotic conjugates: exploiting iron uptake to deliver drugs into bacteria. Clin Microbiol Infect 24:801-802. https://doi.org/10.1016/j.cmi.2018.03.037

12. Ribeiro M, Simões M (2019) Advances in the antimicrobial and therapeutic potential of siderophores. Environ Chem Lett. https:// doi.org/10.1007/s10311-019-00887-9

13. Abdul-Mutakabbir JC, Alosaimy S, Morrisette T et al (2020) Cefiderocol: a novel siderophore cephalosporin against multidrugresistant gram-negative pathogens. Pharmacotherapy 40:12281247. https://doi.org/10.1002/phar.2476

14. Osorio CR, Rivas AJ, Balado M et al (2015) A transmissible plasmid-borne pathogenicity island confers piscibactin biosynthesis in the fish pathogen Photobacterium damselae subsp. piscicida. Appl Environ Microbiol 81:5867-5879. https://doi.org/10.1128/ AEM.01580-15

15. Souto A, Montaos MA, Rivas AJ et al (2012) Structure and biosynthetic assembly of piscibactin, a siderophore from Photobacterium damselae subsp. piscicida, predicted from genome analysis. European J Org Chem 2012:5693-5700. https://doi.org/10.1002/ ejoc. 201200818

16. Balado M, Lages MA, Fuentes-Monteverde JC et al (2018) The siderophore piscibactin is a relevant virulence factor for Vibrio anguillarum favored at low temperatures. Front Microbiol 9:1766. https://doi.org/10.3389/fmicb.2018.01766

17. Thode SK, Rojek E, Kozlowski M et al (2018) Distribution of siderophore gene systems on a Vibrionaceae phylogeny: database searches, phylogenetic analyses and evolutionary perspectives. PLoS ONE 13:1-21. https://doi.org/10.1371/journal.pone.01918 60

18. Drechsel H, Stephan H, Lotz R et al (1995) Structure elucidation of yersiniabactin, a siderophore from highly virulent Yersinia strains. Liebigs Ann. https://doi.org/10.1002/jlac.1995199510243

19. Soengas RG, Anta C, Espada A et al (2006) Structural characterization of vanchrobactin, a new catechol siderophore produced by the fish pathogen Vibrio anguillarum serotype O2. Tetrahedron Lett 47:7113-7116. https://doi.org/10.1016/j.tetlet.2006.07.104

20. Lemos ML, Osorio CR (2010) Iron uptake in Vibrio and Aeromonas. In: Cornelis P, Andrews SC (eds) Iron uptake and homeostasis in microorganisms. Caister Academic Press, Norfolk, pp $117-141$

21. Osorio CR, Juiz-Rio S, Lemos ML (2006) A siderophore biosynthesis gene cluster from the fish pathogen Photobacterium damselae subsp. piscicida is structurally and functionally related to the Yersinia high-pathogenicity island. Microbiology 152:3327-3341. https://doi.org/10.1099/mic.0.29190-0

22. Schwyn B, Neilands JBB (1987) Universal chemical assay for the detection and determination of siderophores. Anal Biochem 160:47-56. https://doi.org/10.1016/0003-2697(87)90612-9

23. Andrews SC, Robinson AK, Rodriguez-Quinones F (2003) Bacterial iron homeostasis. FEMS Microbiol Rev 27:215-237
24. Naikare H, Butcher J, Flint A et al (2013) Campylobacter jejuni ferric-enterobactin receptor CfrA is TonB3 dependent and mediates iron acquisition from structurally different catechol siderophores. Metallomics 5:988. https://doi.org/10.1039/c3mt20254b

25. Wyckoff EE, Allred BE, Raymond KN, Payne SM (2015) Catechol siderophore transport by Vibrio cholerae. J Bacteriol 197:2840 2849. https://doi.org/10.1128/JB.00417-15

26. Ghysels B, Ochsner U, Möllmann U et al (2005) The Pseudomonas aeruginosa pirA gene encodes a second receptor for ferrienterobactin and synthetic catecholate analogues. FEMS Microbiol Lett 246:167-174. https://doi.org/10.1016/j.femsle. 2005.04.010

27. Rey-Varela D, Cisneros-Sureda J, Balado M et al (2019) The outer membrane protein FstC of Aeromonas salmonicida subsp. salmonicida acts as receptor for amonabactin siderophores and displays a wide ligand plasticity. Structure-activity relationships of synthetic amonabactin analogues. ACS Infect Dis 5:1936-1951. https://doi.org/10.1021/acsinfecdis.9b00274

28. Chen J, Forsyth CJ (2003) Synthesis of the apratoxin 2,4-disubstituted thiazoline via an intramolecular aza-Wittig reaction. Org Lett 5:1281-1283. https://doi.org/10.1021/o10342148

29. De la Fuente MC, Segade Y, Valderrama K et al (2021) Convergent total synthesis of the siderophore piscibactin as its Ga3+ complex. Org Lett 23:340-345. https://doi.org/10.1021/acs.orgle tt.0c03850

30. Crosa JH, Walsh CT (2002) Genetics and assembly line enzymology of siderophore biosynthesis in bacteria. Microbiol Mol Biol Rev 66:223-249

31. Ruiz P, Balado M, Fuentes-Monteverde JC et al (2019) The fish pathogen Vibrio ordalii under iron deprivation produces the siderophore piscibactin. Microorganisms 7:313. https://doi.org/ 10.3390/microorganisms 7090313

32. Dubert J, Barja JL, Romalde JL (2017) New insights into pathogenic Vibrios affecting bivalves in hatcheries: present and future prospects. Front Microbiol 8:762. https://doi.org/10.3389/fmicb. 2017.00762

33. Valderrama K, Balado M, Rey-Varela D et al (2019) Outer membrane protein FrpA, the siderophore piscibactin receptor of Photobacterium damselae subsp. piscicida, as a subunit vaccine against photobacteriosis in sole (Solea senegalensis). Fish Shellfish Immunol 94:723-729. https://doi.org/10.1016/j.fsi.2019.09. 066

34. Noël S, Guillon L, Schalk IJ, Mislin GLA (2011) Synthesis of fluorescent probes based on the pyochelin siderophore scaffold. Org Lett 13:844-847. https://doi.org/10.1021/ol1028173

Publisher's Note Springer Nature remains neutral with regard to jurisdictional claims in published maps and institutional affiliations.

\section{Authors and Affiliations}

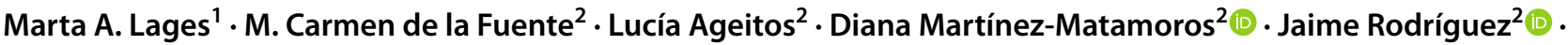 Miguel Balado ${ }^{1}\left[\right.$. Carlos Jiménez $z^{2}(1) \cdot$ Manuel L. Lemos ${ }^{1}$}

Jaime Rodríguez

jaime.rodriguez@udc.es

$\triangle$ Miguel Balado miguel.balado@usc.es

$\checkmark$ Carlos Jiménez carlos.jimenez@udc.es
1 Departamento de Microbiología y Parasitología, Instituto de Acuicultura, Universidade de Santiago de Compostela, 15782 Santiago de Compostela, Spain

2 Departamento de Química, Facultade de Ciencias, Centro de Investigacións Científicas Avanzadas (CICA), Universidade da Coruña, 15071 A Coruña, Spain 